\title{
Visualizing Road Appearance Properties in Driving Video
}

\author{
Zheyuan Wang, Jiang Yu Zheng, Mehmet Kilicarslan \\ Dept. of Computer Science. \\ Indiana University Purdue University Indianapolis \\ Indianapolis, USA
}

\begin{abstract}
- with the increasing videos taken from driving recorders on thousands of cars, it is a challenging task to retrieve these videos and search for important information. The goal of this work is to mine certain critical road properties in a large scale driving video dataset for traffic accident analysis, sensing algorithm development, and testing benchmark. Our aim is to condense video data to compact road profiles, which contain visual features of the road environment. By visualizing road edge and lane marks in the feature space with the reduced dimension, we will further explore the road edge models influenced by road and off-road materials, weather, lighting condition, etc.
\end{abstract}

Keywords-component; Driving video; visualization; data compression; road environment, sensing, illumination

\section{INTRODUCTION}

With the increasing video cameras mounted on vehicles for driving record, driving video becomes available for traffic analysis, driver behavior understanding, and environment monitoring. A lot of information about potential accidents, road infrastructure, and traffic events are worth investigation through mining a large collection of driving video. The objective of this work is to extract common properties of roads in contrast to roadside scenes for developing road sensing and recognition systems in automatic driving. It also serves as a benchmark for vehicle testing to avoid road departure.

Road departure crashes account for more than 15,000 fatalities in the US annually, which is nearly half of all fatalities. Road departure is a major concern while designing driver assistant and warning systems. For autonomous driving, road detection is also a key function. Lane mark detection so far has been widely used in vehicle control. For roads without clear lane marks, however, vision is still the best way to avoid road departure. Finding a road edge to keep the vehicle within road is a much harder task than detecting lane marks with uniformed color and geometry. Although the appearance model of road edges can be generated through graphics simulation, the parameters of material, reflectance, season, lighting, and camera sensitivity have large variations in the real driving. The roads further vary from state to state. The inverse estimation of so many parameters from video is difficult.

Current vision systems tackles the road recognition to a certain degree [3,5]. Research is still going on to implement road detection on special circumstances like raining and fog [2]. However, a single rule cannot handle all the roads because of the changes in road appearance, as shown in
Figure 1. Data classification based on machine learning so far has not covered large datasets. The detection results are dependent on small training data.
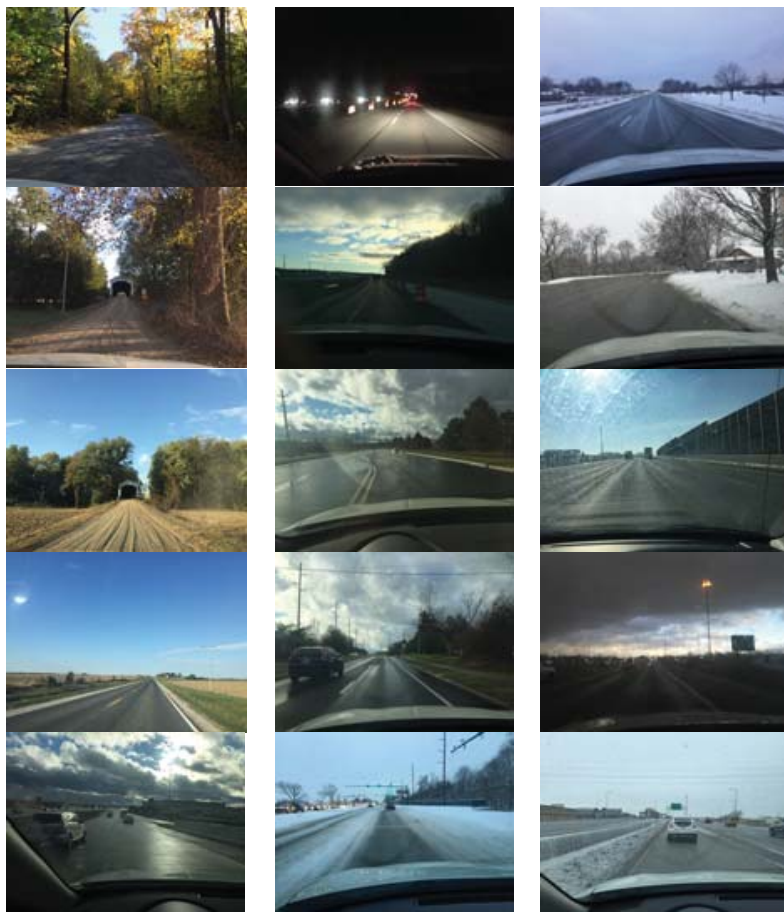

Figure 1. Various road scenes visible in video. Road edges have more drastic changes than the material combinations.

In the intelligent vehicle research, a high accuracy is required in road detection to ensure the driving safety. Analysis of road types based on large samples is necessary. Our goal in this work is to explore how roads vary in appearance under different illumination conditions, how to develop methods for intelligent sensing, and what the most visible road scenes are for vehicle testing. We will first develop a compact representation to condense videos to road profile images, and then compute visual properties from road profiles.

In the following, Section II will describe background model of road edges. Section III proposes the data reduction scheme of video, i.e., the road profile. Section IV computes parameters in profiles and displays them in feature spaces.

\section{ROAD APPEARANCE MODEL}

\section{A. Various Appearances of Road Edge}

Roads show edges that divide road surface and off-

This is the author's manuscript of the article published in final edited form as: 
road regions. The vehicle borne cameras obtain visual data of on-road and off-road appearance according to following physical properties and lighting conductions.

\section{1) Material, Reflectance and Seasonal Factor}

The material of road surface ranges from asphalt, concrete, to gravel. Roadside can be concrete, gravel, brick, soil, grass, vegetation, water, snow, guardrail, construction cones, etc. Their combinations contain more cases. Even for an asphalt surface, it looks dark for new pavement or on wet road, while becomes gray and white after years or in a sunny day with specular reflection of certain degree. A uniformed color on road surface will not occur at repaired spots and shadow areas. In different seasons, off-road scenes can change largely from green to yellow, or even white due to snow coverage. These material differences are described by surface reflectance. The subdivision of materials are further listed in Table I. We denote materials as $m_{l}, m_{2}, \ldots m_{i}$, and their global frequency or probability, $\operatorname{Prob}\left(m_{i}\right)$, in a country like US can be obtained from separate data sources such as GIS database.

TABLE I. SUBDIVISION OF MATERIALS AFFACTED By SEASONS

\begin{tabular}{|l|l|}
\hline Material & Season and status \\
\hline Asphalt & New, old, wet, dry \\
\hline Grass & Green, yellow \\
\hline Vegetation & Green, yellow, gray \\
\hline Snow & Fully covered, whitened \\
\hline Water & Wet, mirror reflection \\
\hline
\end{tabular}

\section{2) Weather, Lighting Condition and Direction}

The road color captured by a camera is also determined from illumination. The light can be directional sunlight in sunny day, diffused light in cloudy day, ambient light in shadow area, dark lit at dusk, and night illumination by vehicle headlights and street lights.

In driving video, cameras may capture direct illuminants during sun rise or sun set. Mostly cameras observe reflected light from scenes, which further include diffused reflection and specular reflection. Specular reflection occurs slightly on road surface when a car faces the sun, or the road surface has water in raining day; the surface will be brightened by reflecting sky or darkened due to high objects like mountains, buildings and trees.

\section{3) Camera Receptance or Sensitivity}

Image intensity in video is not only affected by the camera aperture, but also influenced from its sensitivity in fog, rain, and dirty windshield. Auto-exposure function is used to balance color in the entire field of view during video capturing. Because of the wide field of view of cameras, the driving videos captured are stable in their intensity changes.

\section{B. Data Based Vision Approach}

There has no classification method to detect all kinds of road edges; most algorithms target a certain type of roads. The road segmentation has been using color, intensity, hue, continuity of edges, homogeneity of road surface, etc. However, the thresholds for classification are hard to estimate. Because the combination of materials and lighting can yield hundreds of different cases, not all of them are visually distinct. There should have some clusters in which roads have similar visual characteristics. Big data mining of driving video can sort out these characteristics. This is the first work starting to investigate a full spectrum of visual appearances on roads in contrast to off-road scenes. Several visual features we examine are suitable for real-time road edge detection, which are listed as $V=\{$ on-road and off-road colors, edge contrast, linearity of road edges, homogeneity on road surface . The probability distribution of $V, \operatorname{Prob}(V)$, can be found through sampling millions of locations from driving videos.

Our video data are tagged briefly according to physical categories as described in Table I through a user interface. We can obtain probability of visual features for each road type, $\operatorname{Prob}\left(V / m_{i}\right)$, during the feature collection. Overall, visual appearances have conditional probability as

$$
\operatorname{Prob}(V)=\sum_{\mathrm{i}} \operatorname{Prob}\left(V / m_{i}\right) \operatorname{Prob}\left(m_{i}\right)
$$

with $\operatorname{Prob}\left(m_{i}\right)$ from other resources such as GIS system.

In the driving video, the observing vehicles can change lanes and turn sharply at street crossing, which should be excluded from the road edges. Stopping periods exist while waiting signals and in traffic jams. There have other vehicles around during driving. These should be excluded from the road side scenes. It is well known that white and yellow lane marks provide a much stronger hint of road than any road edge in road recognition. We thus select rural road without clear lane marks or even remove lane marks for visual property collection at road edges. The roads in residential areas are also selected due to lack of lane marks on such roads. The road edge detection algorithm will take the maximum win approach in favor of the result from lane mark detection if both land mark and road edge are visible.

\section{PROFILE FROM DIRVING VIDEO}

A big challenge in video data mining is to deal with the huge data size. We use a compact representation called road profile [1] to visualize road environment in a long 2D image. It reduces data to a line from each frame $(1280 \times 720$ pixels $)$ and a video clip is thus condensed to an image (data reduced to $1 / 720$ ). We focus on a depth of $10-15 \mathrm{~m}$ ahead of vehicles, which is the distance possible to avoid road departure accidents in one or two seconds after the road edge detection in video at $30 \mathrm{~Hz}$.

For each fixed camera pose on a vehicle, the horizon in the video frame is stable except on a rolling road. A horizontal sampling line, $\boldsymbol{L}$, can be set on road to scan the road surface and its projection to the video frame, $I(x, y, t)$, is denoted as $l$, where $t$ is the frame number and $x, y$ are the image coordinates (Fig. 2a). It can cover the driving lane and a part of off-road scenes such as lawn, sidewalk, forests, field, etc. The color pixels on the line are sampled at consecutive $t$ frames and copied to the profile image $P(x, t)$ as shown in Fig. 2b. We sample the video at $30 \mathrm{~Hz}$ and the resulting $P(x, t)$ has the height $T$ as the video length in frame number. The sampling with the pixel line skips some interval on road depending on the vehicle speed. 


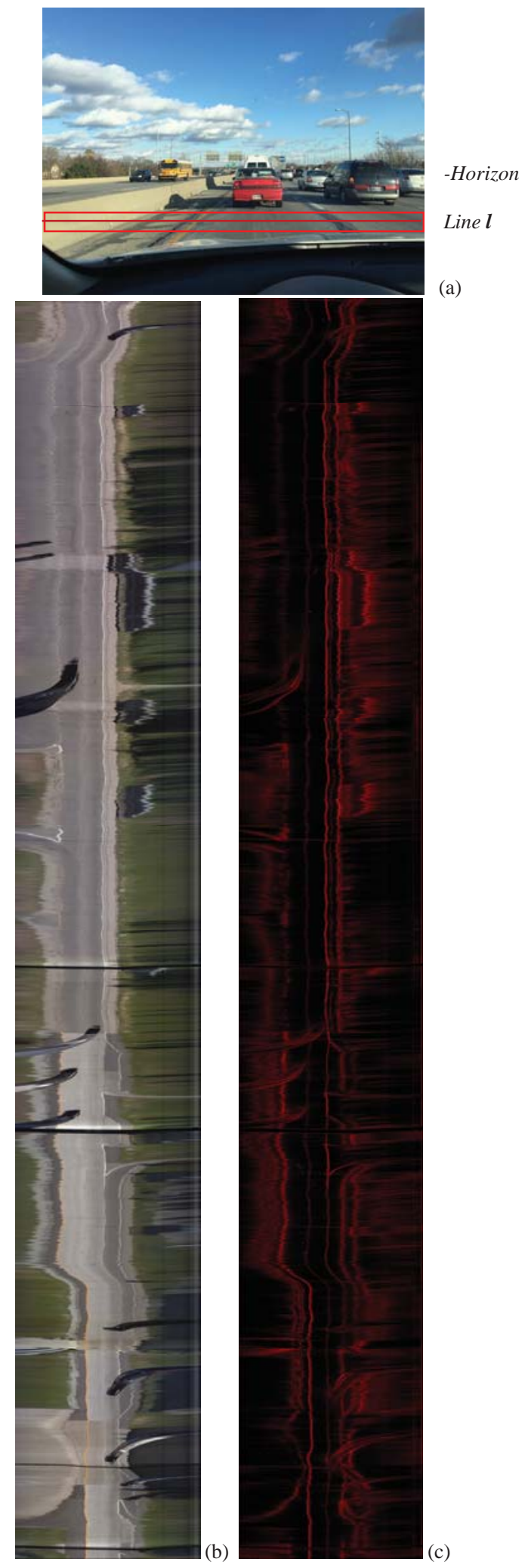

Figure 2 A road profile obtained from a sampling line at $10-15 \mathrm{~m}$ ahead of vehicle. (a) An associated image region around the sampling line is also located for obtaining spatial property of road such as linearity of road edges. (b) The resulting road profile is displayed in right column. (c) The linearity is also displayed in red intensity. The horizontal axes are $\mathrm{x}$ and vertical axes are frame number $t$ upward for both (b) and (c).
If a car keeps driving in a lane, the lane in the video and profile are approximately maintained at the same positions. Because of the vehicle shaking in its fast motion mainly in roll and yaw, the lane marks and road edges are waved in the profile. This waving is more significant at the peripheral of the image and profile. Nevertheless, we still can observe the lanes, road surface, shadow, and the passing vehicles at side lanes. The road profile covers an entire route. Even if the vehicle moves on a curved road, the road profile shows a straight lane in the image. This avoids complex geometry computation of road structure like road curvature, which is an important factor that causes road departure [4], and allows us to focus on color appearance study. In Figure $2 b$, we can observe gray road surface and green lawn at side. Guardrails are visible from place to place in the green area. Some dark patterns initiated on the road surface are the traces of vehicles passing by. The dark horizontal regions spanning over the entire road are the shadow under bridges across the road, and such a dark region is followed by a bright region immediately due to the auto-exposure function of the camera when the vehicle moves out of the shadow area.

\section{FEATURES EXTRACTED FROM RoAd PROFILE}

The road features such as colors on-road and off-road, contrast at edge are captured in the profile for each location $t$. For colors, the hue, saturation, and intensity in the road and off-road areas are also calculated. We further register the linearity of edges and believe that a long edge provides a strong clue of road margin, as compared to isolated spots with high contrast like potholes and repaired marks.

The computation of linearity is within a belt around the sampling line in each frame as shown in Figure 2(a). The result linearity, $L(x)$, indicates how long and strong an edge is in the image through position $x$ on the sampling line. For this purpose, we extract gradient in the belt with orientation and magnitude. Its extension along the orientation direction intersects sampling line $\boldsymbol{l}$ at an intercept position. The linearity value at position $x$ is thus accumulated from the gradient values of all the edge points through it by extension. A long road edge will have many edge points voting toward their intersection on the sampling line. The linearity thus has clear peaks at the road edge position, lane marks, and other linear structure on the road. The linearity values from each frame are stored in $L(x, t)$ for frame $t$ and the linearity of entire road is stored in an image as shown in Figure 2(c), in addition to Fig. 2(b). A lane mark position has strong linearity in the frame even if it is waved in the road profile.

Another property to compute is the homogeneity of road surface. Because the road is paved in certain material for passing, the homogeneity is simply gradient values on the sampling line, which involves several pixels around the line in its computation. This is registered in another image $H(x, t)$.

We further extract prominent parameters at each position on road by averaging road profile values along $x$. Road properties are then described by a tuple $V(t)=\left\{C_{o n}(t), C_{o f f}(t)\right.$, $C(t), L(t), H(t)\}$, where $C_{o n}$ and $C_{o f f}$ are color on and off road, which contain three $\mathrm{R}, \mathrm{G}, \mathrm{B}$ values. This data reduction from the road profile to temporal sequence $V(t)$ allows the 
processing of long video sequences.

To sort the properties of different roads, we visualize the feature space $V(t)$ to explore the possibility for road classification. Several plots of feature distributions are designed for long roads according to their physical categories as shown in Figure 3. In the plot of $V(t)$, a feature point in the 2D space has an intensity indicating the frequency of road edge with such feature properties. Combined with data mining methods, this visualization will explore and confirm the clustering of road edges in the video database.
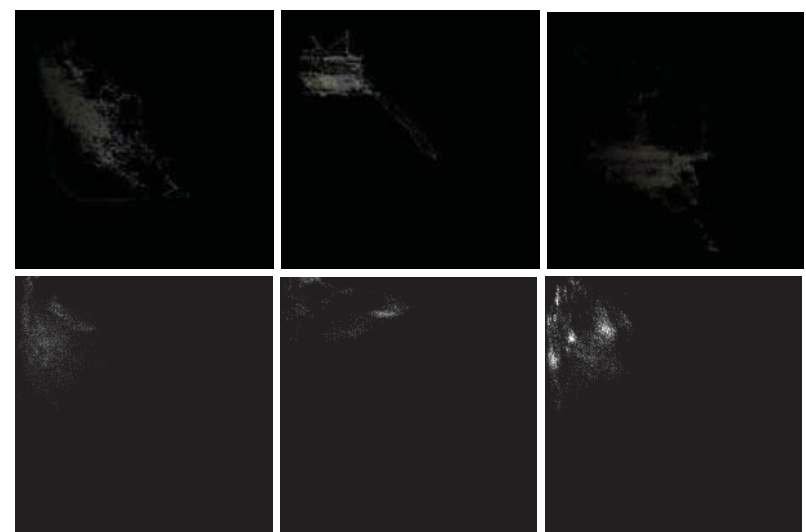

Figure 3. Road edge color distribution on two sides for three driving videos. (Top row) the horizontal axes are intensity of $\mathrm{C}_{\text {off }}$ and vertical axes downward are intensity of $\mathrm{C}_{\mathrm{on}}$. (Bottom row) the vertical axes downward are Intensity $_{\text {off }}-$ Intensity $_{\text {on }}$ and the horizontal axes are the saturation.

\section{EXPERIMENTS}

We have experimented various roads with driving video. In a sunny day, there are many shadow regions on road when a car moves into tree areas (Figure 4c). That is, a road profile may contain multiple types of roads. The feature spaces may be plotted with multiple clusters when the road length increases. Also, it is interesting to find that the night roads in rural area with insufficient illumination are purely followed according to lane marks under vehicle headlight (Figure 4d).

In the visualization, we found that intensity is the most important factor to separate road edges, since it can provide differences between on-road and off-road even if the illumination is dark. Saturation is the second significant factor to separate regions on two sides of road edge (Fig. 4b).

\section{CONCLUSION}

This work targets at visualizing road edge features for road sensing using driving video. Several efforts have been made to largely condense the data size of video to a sequence of feature vectors of the road length, which is suitable for big video data mining. The road profile includes information for road edge detection in autonomous and safety driving. Some important conclusion is expected to be found through the visualization and big data mining.

\section{REFERENCES}

[1] M. Kilicarslan, J. Y. Zheng, "Temporal video profile from driving video," IEEE Symposium on Intelligent Vehicles, pp. 529-551, 2014.

[2] N. Gimonet, A. Cord, G. Saint Pierre, How to predict real road state from vehicle embedded camera? IEEE Symposium on Intelligent
Vehicles 2015, 593-598

[3] Tobias Kuhnl and Jannik Fritsch, Visio-spatial road boundary detection for unmarked urban and rural roads, IEEE Symposium on Intelligent Vehicles 2014, 1251-1256.

[4] A. B. Hillel, R. Lerner, D. Levi, and G. Raz, (2014). Recent progress in road and lane detection: a survey. Machine Vision and Applications, 25(3), 727-745

[5] C. Guo and S. Mita, Semantic-based road environment recognition in mixed traffic for intelligent vehicles and advanced driver assistance systems, 15th Int. IEEE Conf. Intelligent Transportation Systems, 444-450, 2012

[6] A. Jazayeri, H Cai, J. Y. Zheng, M Tuceryan, Vehicle detection and tracking in car video based on motion model, Intelligent Transportation Systems, IEEE Transactions on 12 (2), 583-595, 2011.

[7] S. Wang, S. Luo, Y. Huang, J. Y. Zheng, P. Dai, Q. Han, Railroad online: acquiring and visualizing route panoramas of rail scenes, The Visual Computer 30 (9), 1045-1057.

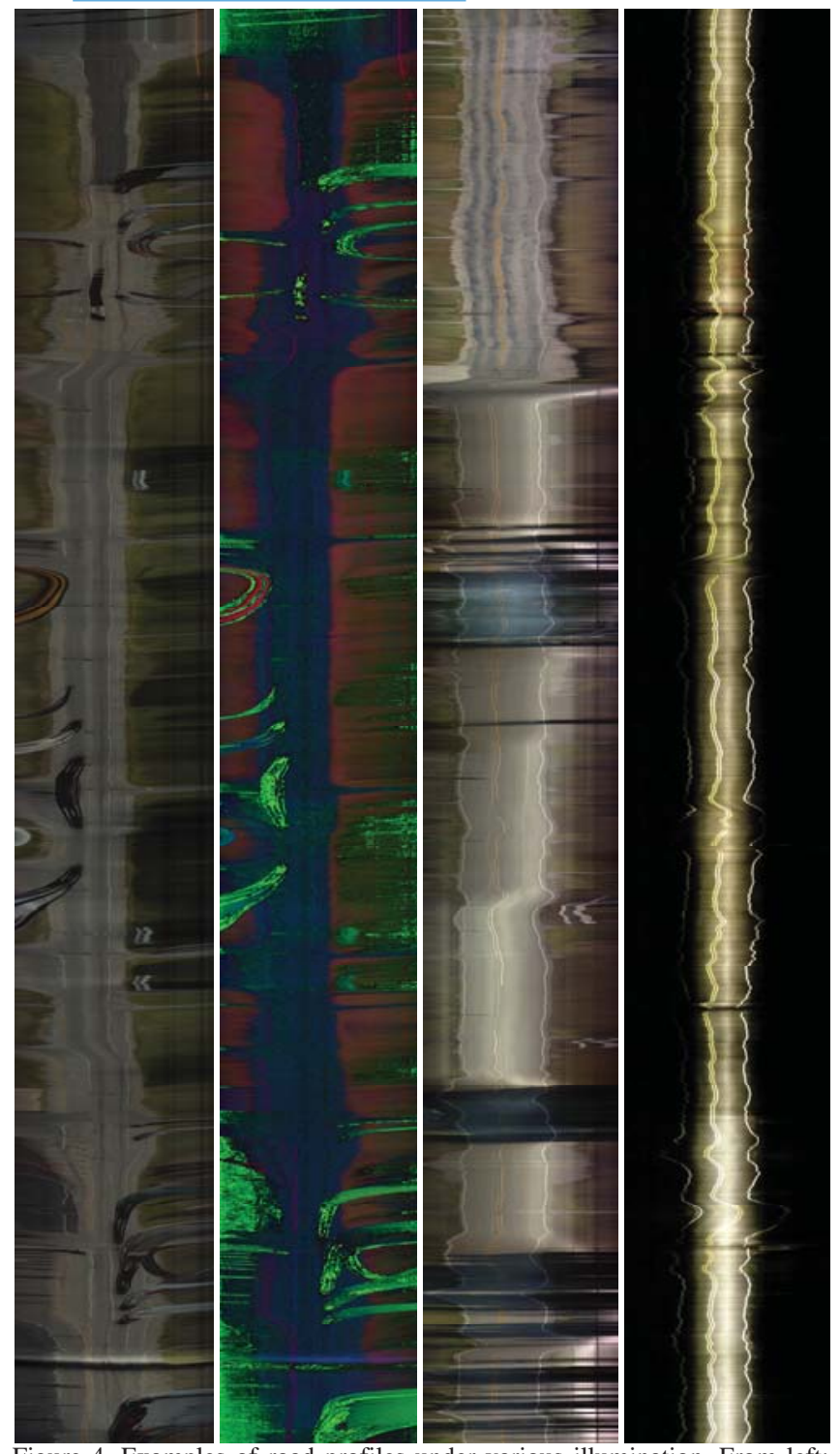

Figure 4. Examples of road profiles under various illumination. From left: (a) Cloudy day. Later segment of road has no lane marks in a residential area, (b) HIS Space display of (a) in three channels S, H, and I. At region without color, hue values are unstable (displayed in green). (c) Sunny day with shadow regions. (d) Road profile with road lit by vehicle headlight in night driving. 